\title{
Beauty to open charm final states at LHCb
}

\section{Fionn Bishop, on behalf of the LHCb collaboration ${ }^{a, *}$}

${ }^{a}$ Cavendish Laboratory, University of Cambridge

19 J J Thomson Avenue, Cambridge, United Kingdom

E-mail: fionn.caitlin.ros.bishop@cern.ch

The latest studies of beauty meson decays to open charm final states using data collected by LHCb are reviewed. The presented results include new branching fraction measurements using data collected during Run 1 and Run 2. These decay modes can, either directly or as inputs to other analyses, improve constraints on the Cabibbo-Kobayashi-Maskawa (CKM) quark-mixing matrix. The presented branching fraction measurements are also useful for improving our understanding of hadronic $B$ decays.

*** The European Physical Society Conference on High Energy Physics (EPS-HEP2021), *** *** 26-30 July $2021 * * *$

*** Online conference, jointly organized by Universität Hamburg and the research center DESY ***

\footnotetext{
${ }^{*}$ Speaker
} 


\section{Introduction}

The unitarity of the Cabibbo-Kobayashi-Maskawa (CKM) matrix forms an excellent test of Beyond Standard Model (BSM) Physics. This test requires an improved experimental precision on CKM parameters, which is one of the main goals of the LHCb experiment [1]. Measurements of beauty to open charm decays are used to determine certain CKM parameters. Furthermore, since it is challenging to make predictions for fully hadronic decays, branching fraction predictions can vary substantially between theory methods [2]. This motivates the study of beauty to open charm decays, especially for rare and heavy particles like the $B_{c}^{+}$meson, as a probe of QCD in hadronic decays.

\section{Updated search for $B_{c}^{+}$decays to two charm mesons}

$B_{c}^{+}$decays to two charm mesons, in particular where one meson is a $D_{s}^{(*)+}$ (Fig. 1), are expected to have large, $O(1), \mathrm{CP}$ asymmetries. This allows $B_{c}^{+} \rightarrow D D$ decays to provide sensitivity to the CKM phase $\gamma$ [3]. Furthermore, predictions on the branching fractions vary by an order of magnitude [2] so branching fraction measurements could constrain understanding of the $B_{c}^{+}$meson. Observation of these decays is challenging, primarily due to the small production cross section of the $B_{c}^{+}$meson. A previous search performed in data corresponding to $3 \mathrm{fb}^{-1}$ of $p p$ collisions found no evidence of signal [4]. An updated search has now been performed in data corresponding to $9 \mathrm{fb}^{-1}[5]$.

The search is performed in six channels: $D_{s}^{+} \stackrel{(-)}{D^{0}}, D^{+} \stackrel{(-)}{D}^{0}$, and $D^{*+} D^{0}$. In each channel the search is for both the fully reconstructed $B_{c}^{+}$decay and partially reconstructed $B_{c}^{+}$decays where one or more excited charm mesons emit a soft neutral particle which is not reconstructed. The branching fraction is measured relative to that of the abundant $B^{+} \rightarrow D \bar{D}^{0}$ decay:

$$
\mathcal{R}=\frac{f_{c}}{f_{u}} \frac{\mathcal{B}\left(B_{c}^{+} \rightarrow D D\right)}{\mathcal{B}\left(B^{+} \rightarrow D \bar{D}^{0}\right)}=\frac{N_{B_{c}^{+}}}{N_{B^{+}}} \frac{\varepsilon_{B^{+}}}{\varepsilon_{B_{c}^{+}}}
$$

where $\frac{f_{c}}{f_{u}}$ is the ratio of $B_{c}^{+}$to $B^{+}$fragmentation fractions, $N_{B_{(c)}^{+}}$are the yields and $\varepsilon_{B_{(c)}^{+}}$are the selection efficiencies.

Preselection requirements are applied and then a boosted decision tree (BDT) is trained to discriminate between signal and random combinations of tracks. The lowest purity data according

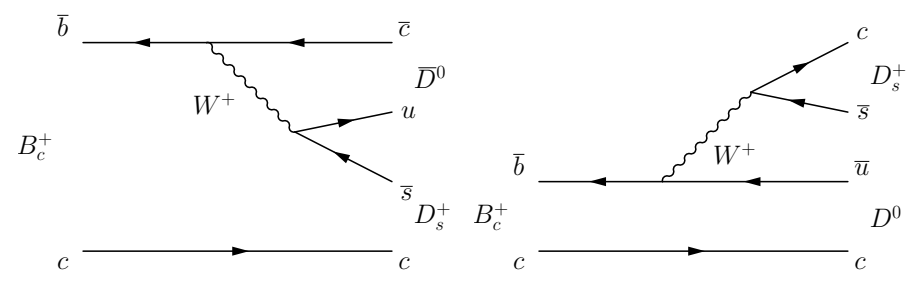

Figure 1: (Left) The Cabbibo-favoured, colour-suppressed diagram which dominates $B_{c}^{+} \rightarrow D_{s}^{+} \bar{D}^{0}$ decays and (right) the Cabbibo-suppressed, colour-favoured diagram which dominates $B_{c}^{+} \rightarrow D_{s}^{+} D^{0}$ decays. 

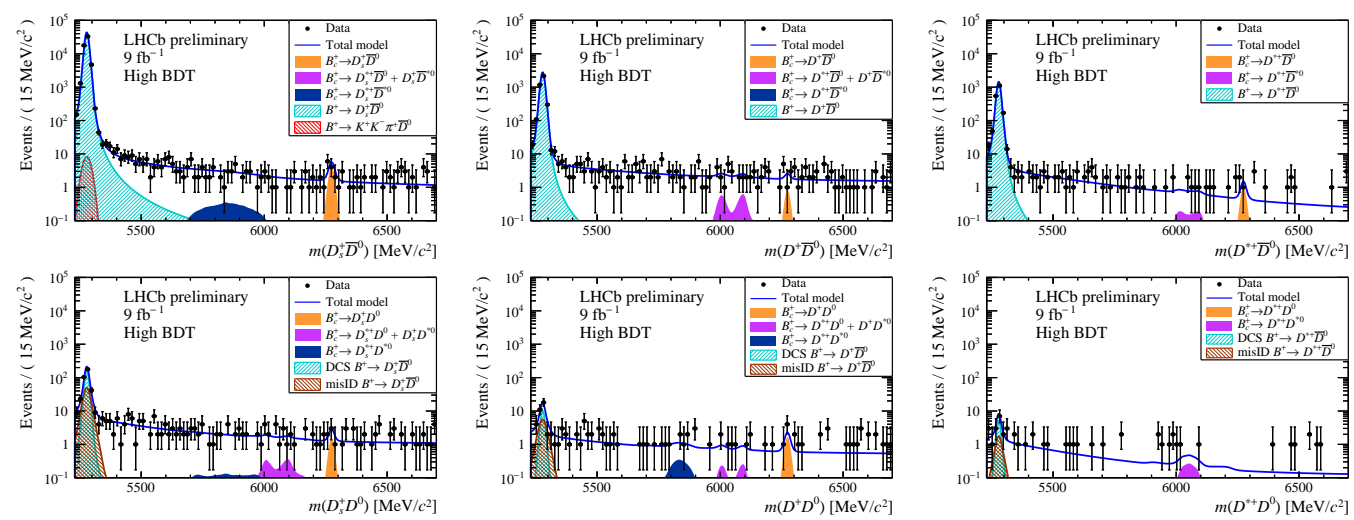

Figure 2: B mass distributions of selected candidates in the highest BDT response samples in the (top left) $D_{s}^{+} \bar{D}^{0}$, (bottom left) $D_{s}^{+} D^{0}$, (top centre) $D^{+} \bar{D}^{0}$, (bottom centre) $D^{+} D^{0}$, (top right) $D^{*+} \bar{D}^{0}$, and (bottom right) $D^{*+} D^{0}$ channels. The fitted model is overlaid.

to the BDT response is discarded. To enhance sensitivity to signal, the remaining data is divided into three subsamples in BDT response. The branching fraction ratio $\mathcal{R}$ is found in a simultaneous maximum likelihood fit of a model to the data (Fig. 2).

The branching fraction $\mathcal{B}\left(B_{c}^{+} \rightarrow D_{s}^{+} \bar{D}^{0}\right)=\left(3.5_{-1.2-0.2}^{+1.5+0.3} \pm 1.0\right) \times 10^{-4}$ is measured where the uncertainties are from statistical, systematic and external sources respectively. The significance of the associated signal is $3.4 \sigma$. This constitutes the first evidence of $B_{c}^{+} \rightarrow D_{s}^{+} \bar{D}^{0}$ and the measured branching fraction is in tension with predictions by two orders of magnitude. No other evidence of $B_{c}^{+} \rightarrow D D$ decays is observed. Upper limits are set on the branching fraction of fourteen $B_{c}^{+} \rightarrow D D$ decays, including six fully reconstructed and eight partially reconstructed decays.

\section{Observation of the $B_{s}^{0} \rightarrow D^{* \pm} D^{\mp}$ decay}

The $B^{0} \rightarrow D^{* \pm} D^{\mp}$ decay is dominated by tree and penguin diagrams which provide access to the CKM phase $\beta$. Subleading contributions are from W-exchange and penguin-annihilation diagrams, which are the dominant contributions to $B_{s}^{0} \rightarrow D^{* \pm} D^{\mp}$ (Fig. 3). Therefore a measurement of the $B_{s}^{0} \rightarrow D^{* \pm} D^{\mp}$ branching fraction is useful in determining the size of the subleading contributions to $B^{0} \rightarrow D^{* \pm} D^{\mp}[6]$.

A search for the $B_{s}^{0} \rightarrow D^{* \pm} D^{\mp}$ decay is performed in data corresponding to $9 \mathrm{fb}^{-1}$ of $p p$ collisions collected between 2011 and 2018 [7]. Preselection, including on the response of a BDT trained to discriminate against random combinations of tracks, is applied. The yields of the
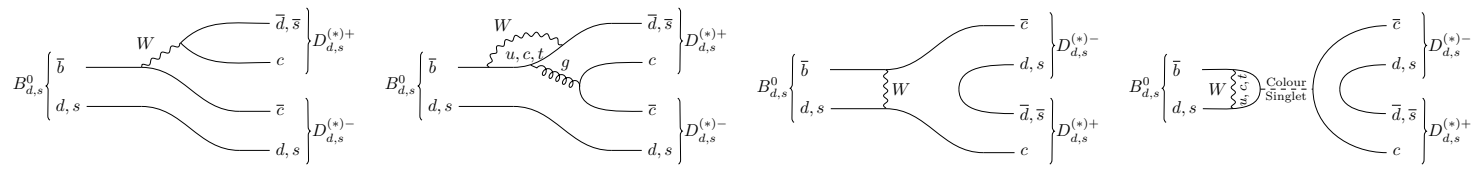

Figure 3: (Starting from left) Tree and penguin diagrams contributing to $B^{0} \rightarrow D_{(s)}^{(*) \pm} D^{(*) \mp}$ and $B_{s}^{0} \rightarrow D_{(s)}^{(*) \pm} D_{s}^{(*) \mp}$ decays and W-exchange and penguin-annihilation diagrams contributing to $B_{(s)}^{0} \rightarrow D_{(s)}^{(*)+} D_{(s)}^{(*)-}$ decays. 

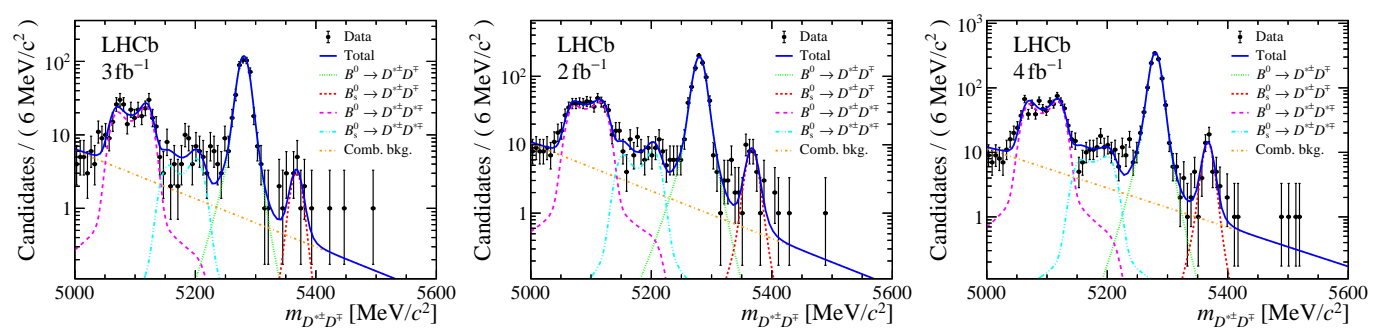

Figure 4: $D^{* \pm} D^{\mp}$ mass distributions in (left) 2011-12, (centre) 2015-16 and (right) 2017-18 data and the corresponding fits.

$B_{s}^{0} \rightarrow D^{* \pm} D^{\mp}$ and $B^{0} \rightarrow D^{* \pm} D^{\mp}$ decays are determined from a maximum likelihood fit to the $D^{* \pm} D^{\mp}$ mass distribution. The fits are shown in Fig. 4 and the corresponding yields are $12 \pm 4$ and $466 \pm 22$ (2011-12), $34 \pm 7$ and $780 \pm 29$ (2015-16), and $49 \pm 8$ and $1263 \pm 36$ (2017-18).

The branching fraction is measured relative to that of $B^{0} \rightarrow D^{* \pm} D^{\mp}$ by correcting the yield ratio for the ratio of the selection efficiencies $\left(\varepsilon_{B_{s}^{0}} / \varepsilon_{B^{0}}\right)$ and the ratio of the $B_{s}^{0}$ to the $B^{0}$ fragmentation fractions $\left(f_{s} / f_{d}\right)$. The measured branching fraction ratio is

$$
\frac{\mathcal{B}\left(B_{s}^{0} \rightarrow D^{* \pm} D^{\mp}\right)}{\mathcal{B}\left(B^{0} \rightarrow D^{* \pm} D^{\mp}\right)}=\frac{N_{B_{s}^{0}}}{N_{B^{0}}} \frac{\varepsilon_{B^{0}}}{\varepsilon_{B_{s}^{0}}} \frac{f_{d}}{f_{s}}=0.137 \pm 0.017 \pm 0.002 \pm 0.006
$$

where the first uncertainty is statistical, the second is systematic and the third is from the uncertainty on $f_{s} / f_{d}$. This measurement is in agreement with predictions which assume prominent contributions from rescattering [8] but disagrees with predictions from perturbative QCD [9].

\section{Measurement of the branching fraction of the $B^{0} \rightarrow D_{s}^{+} \pi^{-}$decay}

The branching fraction of the $B^{0} \rightarrow D_{s}^{+} \pi^{-}$decay (Fig. 5) provides access to multiple parameters in the CKM matrix. Firstly it is proportional to $\left|V_{u b}\right|^{2}$ :

$$
\mathcal{B}\left(B^{0} \rightarrow D_{s}^{+} \pi^{-}\right)=\Phi\left|V_{u b}\right|^{2}\left|V_{c s}\right|^{2}\left|F\left(B^{0} \rightarrow \pi^{-}\right)\right|^{2} f_{D_{s}^{+}}^{2}\left|a_{\mathrm{NF}}\right|^{2}
$$

where $\Phi$ is a phase-space factor, $F\left(B^{0} \rightarrow \pi^{-}\right)$is the form factor, $f_{D_{s}^{+}}$is the $D_{s}^{+}$decay constant and $a_{\mathrm{NF}}$ encapsulates non-factorisable effects. It furthermore provides access to the ratio $r_{D \pi}$ which is essential for interpreting the CP asymmetries of $B^{0} \rightarrow D^{\mp} \pi^{ \pm}$in terms of the CKM angle $\gamma$ :

$$
r_{D \pi}=\tan \theta_{c} \frac{f_{D^{+}}}{f_{D_{s}^{+}}} \sqrt{\frac{\mathcal{B}\left(B^{0} \rightarrow D_{s}^{+} \pi^{-}\right)}{\mathcal{B}\left(B^{0} \rightarrow D^{-} \pi^{+}\right)}}
$$

where $\theta_{c}$ is the Cabibbo angle and $f_{D^{+}}$is the $D^{+}$decay constant. The branching fraction is measured in data corresponding to $5 \mathrm{fb}^{-1}$ of integrated luminosity collected between 2011 and 2016 [10]. $B^{0} \rightarrow D_{s}^{+} \pi^{-}\left(B^{0} \rightarrow D^{-} \pi^{+}\right)$candidates are formed by combining a $D_{s}^{+} \rightarrow K^{+} K^{-} \pi^{+}$ $\left(D^{-} \rightarrow K^{-} \pi^{+} \pi^{-}\right)$candidate with a pion of opposite charge. Loose preselection is applied to reduce backgrounds, including a BDT trained to discriminate against random combinations of tracks.

The $B^{0} \rightarrow D_{s}^{+} \pi^{-}$and $\bar{B}_{s}^{0} \rightarrow D_{s}^{+} \pi^{-}$yields are extracted in a two-dimensional maximumlikelihood fit to the $D_{s}^{+} \pi^{-}$and the $K^{+} K^{-} \pi^{+}$invariant mass distributions. The $B^{0} \rightarrow D^{-} \pi^{+}$yield 


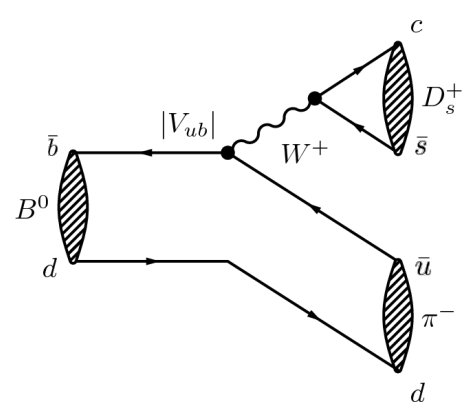

Figure 5: The dominant and only leading order diagram contributing to the $B^{0} \rightarrow D_{s}^{+} \pi^{-}$decay.
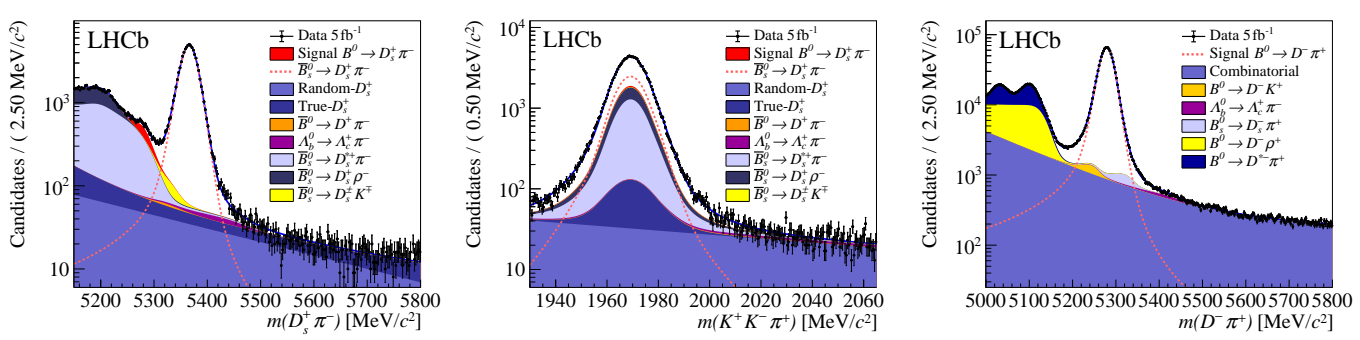

Figure 6: The (left) $D_{s}^{+} \pi^{-}$and (centre) $K^{+} K^{-} \pi^{+}$invariant mass distributions of selected $B \rightarrow D_{s}^{+} \pi^{-}$ candidates and the (right) $D^{-} \pi^{+}$invariant mass distribution of selected $B^{0} \rightarrow D^{-} \pi^{+}$candidates in data corresponding to $5 \mathrm{fb}^{-1}$ of integrated luminosity collected in 2011-2016. Fitted models are overlaid.

is extracted in a maximum-likelihood fit to the $D^{-} \pi^{+}$invariant mass distribution. The sum of the fits to data collected in 2011-12 and 2015-16 are shown in Fig. 6. The corresponding yields are $N_{B^{0} \rightarrow D_{S}^{+} \pi^{-}}=(8.9 \pm 0.8) \times 10^{2}(2011-12)$ and $(1.12 \pm 0.11) \times 10^{3}(2015-16) ; N_{\bar{B}_{s}^{0} \rightarrow D_{s}^{+} \pi^{-}}=(3.370 \pm$ $0.023) \times 10^{4}(2011-12)$ and $(4.647 \pm 0.027) \times 10^{4}(2015-16)$; and $N_{B_{S}^{0} \rightarrow D^{-} \pi^{+}}=(4.971 \pm 0.013) \times 10^{5}$ (2011-12) and (6.294 \pm 0.016$) \times 10^{5}(2015-16)$.

The branching fraction is measured relative to $\mathcal{B}\left(B^{0} \rightarrow D^{-} \pi^{+}\right)$by correcting the yield ratio for the efficiency ratio and $D$ branching fractions. This branching fraction ratio is used to determine $r_{D \pi}=0.0163 \pm 0.0007 \pm 0.0007 \pm 0.0033$ where the first uncertainty is statistical, the second is systematic and the third is from possible non-factorisable $\mathrm{SU}(3)$-breaking effects [11]. Additionally the known $\mathcal{B}\left(B^{0} \rightarrow D^{-} \pi^{+}\right)$is input to obtain $\mathcal{B}\left(B^{0} \rightarrow D_{s}^{+} \pi^{-}\right)=(19.4 \pm 1.8 \pm 1.3 \pm 1.2) \times 10^{-6}$ where the first uncertainty is statistical, the second is systematic and the third is from uncertainty on input branching fractions. This is consistent with the current world average of $(21.6 \pm 2.6) \times 10^{-6}$ and is the most precise single measurement to date [12]. The product $\left|V_{u b}\right|\left|a_{\mathrm{NF}}\right|=(3.14 \pm 0.20 \pm 0.25) \times 10^{-3}$ where the first uncertainty is from the branching fraction measurement and the second from external inputs, is subsequently extracted.

Additionally, the Cabbibo-favoured $B^{0} \rightarrow D^{-} \pi^{+}$and $\bar{B}_{s}^{0} \rightarrow D_{s}^{+} \pi^{-}$yields are used to measure the dependence of $\frac{f_{s}}{f_{d}}$ on the proton-proton collision energy $\sqrt{s}$. Knowledge of $\frac{f_{s}}{f_{d}}$ is vital for extracting the absolute branching fraction of $B_{s}^{0}$ decays, many of which, for example $B_{s}^{0} \rightarrow \mu \mu$, may be sensitive to BSM Physics. $\frac{f_{s}}{f_{d}}$ is proportional to the efficiency-corrected yield ratio

$$
\mathcal{R}=\frac{N_{\bar{B}_{s}^{0} \rightarrow D_{s}^{+} \pi^{-}}}{N_{B^{0} \rightarrow D^{-} \pi^{+}}} \frac{\varepsilon_{B^{0} \rightarrow D^{-} \pi^{+}}}{\bar{B}_{\bar{B}^{0} \rightarrow D_{s}^{+} \pi^{-}}}
$$


The collision energy dependence extracted in a linear fit is $\mathcal{R}=0.156(6)+0.0008(6) \sqrt{s} / \mathrm{TeV}$. This measurement is used as an input to a recent combination of $\frac{f_{s}}{f_{d}}[13]$.

\section{Conclusion}

With the $p p$ collision data collected in Run 1 and Run 2 of the LHC, the LHCb detector has made many important measurements of beauty to open charm decays. The most recent have been discussed in this review. These are useful to improve constraints on the CKM matrix and understanding of fully hadronic decays of $B$ hadrons. An increase in integrated luminosity by the end of Run 3 alongside improvements in trigger efficiencies will greatly enhance Physics sensitivity in this domain.

\section{References}

[1] LHCв collaboration, The LHCb Detector at the LHC, JINST 3 (2008) S08005.

[2] Z. Rui, Z. Zhitian and C.-D. Lu, The Double charm decays of $B_{c}$ Meson in the Perturbative QCD Approach, Phys. Rev. D 86 (2012) 074019 [1203.2303].

[3] M. Masetti, CP violation in Bc decays, Physics Letters B 286 (1992) 160.

[4] LHCв collaboration, Search for B $B_{c}^{+}$decays to two charm mesons, Nucl. Phys. B 930 (2018) 563 [1712.04702].

[5] LHCв collaboration, Updated search for $B_{c}^{+}$decays to two charm mesons, 2109. 00488.

[6] L. Bel, K. De Bruyn, R. Fleischer, M. Mulder and N. Tuning, Anatomy of $B \rightarrow D \bar{D}$ decays, JHEP 07 (2015) 108 [1505. 01361].

[7] LHCв collaboration, Observation of the $B_{s}^{0} \rightarrow D^{* \pm} D^{\mp}$ decay, JHEP 03 (2021) 099 [2012.11341].

[8] M. Gronau, D. London and J.L. Rosner, Rescattering Contributions to rare B-Meson Decays, Phys. Rev. D 87 (2013) 036008 [1211.5785].

[9] Y. Li, C.-D. Lu and Z.-J. Xiao, Rare decays $B^{0} \rightarrow D_{s}^{(*)+} D_{s}^{(*)-}$ and $B^{0} \rightarrow D^{(*)+} D^{(*)-}$ in perturbative QCD approach, J. Phys. G 31 (2005) 273 [hep-ph/0308243].

[10] LHCв collaboration, Measurement of the branching fraction of the $B^{0} \rightarrow D_{s}^{+} \pi^{-}$decay, Eur. Phys. J. C 81 (2021) 314 [2010.11986].

[11] K. De Bruyn, R. Fleischer, R. Knegjens, M. Merk, M. Schiller and N. Tuning, Exploring $B_{s} \rightarrow D_{s}^{(*) \pm} K^{\mp}$ Decays in the Presence of a Sizable Width Difference $\Delta \Gamma_{s}$, Nucl. Phys. $B \mathbf{8 6 8}$ (2013) 351 [1208.6463].

[12] Particle Data Group collaboration, Review of Particle Physics, PTEP 2020 (2020) $083 \mathrm{C} 01$.

[13] LHCв collaboration, Precise measurement of the $f_{s} / f_{d}$ ratio of fragmentation fractions and of $B_{s}^{0}$ decay branching fractions, Phys. Rev. D 104 (2021) 032005 [2103.06810]. 